\title{
Etiology and complications of acute gastroenteritis in hospitalized children
}

\author{
Nardin Elias, Daniela Pop \\ "Iuliu Hatieganu" University of Medicine and Pharmacy, Cluj-Napoca, Romania
}

\begin{abstract}
Diarrheal diseases represent a leading cause of morbidity and mortality in children less than 5 years of age. The aim of our study was to examine the most common etiologies and complications of acute gastroenteritis (AGE) in children hospitalized in our clinic, in different seasons.

Material and method. We retrospectively evaluated the data of 199 children [mean value of the age \pm standard deviation $(S D)=2.45 \pm 2.5$ years] admitted in one year in our department and diagnosed with AGE.

Results. Rotavirus was found in $15.6 \%$ (31/199) of the patients; adenovirus in 9.5\% (19/199). Rotavirus was found to be the dominating confirmed pathogen in most of the months, throughout the year. The duration of the symptoms from the onset of the disease until discharge ranged between 2-21 days, mean value $\pm S D=5.2 \pm 2.69$ days. More than half of the patients had signs of moderate dehydration (53.3\%-106/199 patients) and $39.6 \%(79 / 199)$ signs of mild dehydration.

Conclusions. In our study, AGE affected children between 1-5 years of age primarily. Rotavirus was the leading etiology found in most pediatric age groups. Severe complications in children with AGE and no other chronic diseases were rare.
\end{abstract}

Keywords: gastroenteritis, children, etiology, complications, rotavirus

\begin{abstract}
Abbreviations
AGE - acute gastroenteritis

WHO - World Health Organization

$\mathrm{SD}$ - standard deviation

IV - intravenous

ORS - oral rehydration solutions
\end{abstract}

\section{INTRODUCTION}

Diarrheal diseases represent a leading cause of morbidity and mortality in children less than 5 years of age $(1,2,3)$. In the past four decades, a considerable increase in the newly acknowledged etiological agents of acute gastroenteritis (AGE) has been reached, with more than 20 different microorganisms are recently recognized (4). Many risk factors have been discussed; among the main ones are age (5 years old or younger), malnutrition and other diseases and comorbidities, representing as well factors which may lead to disease escalation (4). Viral pathogens represent the most common etiologies of AGE - rotavirus followed by norovirus $(4,5,6)$. Among some of the bacterial pathogens involved are: Escherichia coli, Campylobacter jejuni, Salmonella spp., and Shigella spp. $(4,5,6)$.
In the United States, rotavirus accounts for $40 \%$ of all diarrheal cases (5), and $36 \%$ of hospitalization in children under the age of 5 worldwide (7). Similar findings are seen in Europe (8) where 55\% of admissions and one-third of emergency visits due to AGE in children under 5, are attributed to rotavirus.

Although diarrhea among children is a widespread problem in both developing and developed countries, it has distinct consequences. In developing countries, gastrointestinal infections are associated with a high rate of mortality where 1 out of 40 children will die due to diarrhea (9). In these low socioeconomic countries, the incidence among infants is 3.8 episodes per child per year, and 2.1 episodes per child per year in older children (1-4 years old) (10). In contrast, in more developed countries, the incidence of acute diarrhea is 1-2 episodes per child per year in children under 3 (4). 
The primary and most important complication of AGE is dehydration, which is also the cause of death in some cases. Other complications include electrolytes disturbances, hypoglycemia, hyperglycemia and metabolic acidosis (4).

The introduction of rotavirus vaccine has led to a reduction in rotavirus associated diarrheal diseases, with the contrary increase in norovirus cases which has become the main cause of addressed AGE cases in the USA associated with considerable financial burden and medical costs (11).

Our study aimed to examine the most common etiologies and complications of AGE in children in different seasons, in the $3^{\text {rd }}$ Pediatric Clinic in ClujNapoca.

\section{MATERIAL AND METHOD}

We conducted the study between October 2017 and September 2018. Our database consists of 199 patients [111- boys (55\%), 88-girls (45\%)], diagnosed with AGE, and divided into four age groups: 0-1 year, 1-5 years, 5-10 years and above 10 years of age.

The data were collected from the patients' files. We collected clinical data (presence of vomiting, diarrhea, number of stools, consistency, presence of pathological products, fever, duration of symptoms, weight, height, signs of dehydration), laboratory investigations (determination of acid-base balance, electrolytes, blood glucose, inflammatory markers, liver enzymes, parameters of the renal function, microscopic stool examination, determination of viral antigens in the stool, cultures) and data regarding the treatment. We excluded from the study patients with chronic diseases such as malnutrition, chronic gastrointestinal disorders, or neurological pathologies, which may affect the course of the disease.
It was a retrospective, descriptive study. For the statistical analysis, we used the Excel program and expressed the results in percentages, mean value \pm standard deviation (SD) and median value. We used T-test to compare some of the results, with a statistically significant value of $\mathrm{p}<0.05$.

\section{RESULTS}

The age range of the patients admitted in our clinic with AGE was between 2 weeks and 14 years, mean value $\pm \mathrm{SD}=2.45 \pm 2.5$ years. The median value of the age was 1.6 years.

The total number of patients included in our study199- was divided under four different age groups. The highest number of patients having AGE, were children between 1-5 years of age, representing more than half $(55.3 \%)$ of the studied population. There were 63 patients aged between 0 and 1 year old (31.7\%), 20 patients aged 5 to 10 years $(10 \%)$ and 6 patients older than 10 years of age $(3 \%)$.

As seen in figure 1, the highest number of AGE children admitted was in November followed by August.

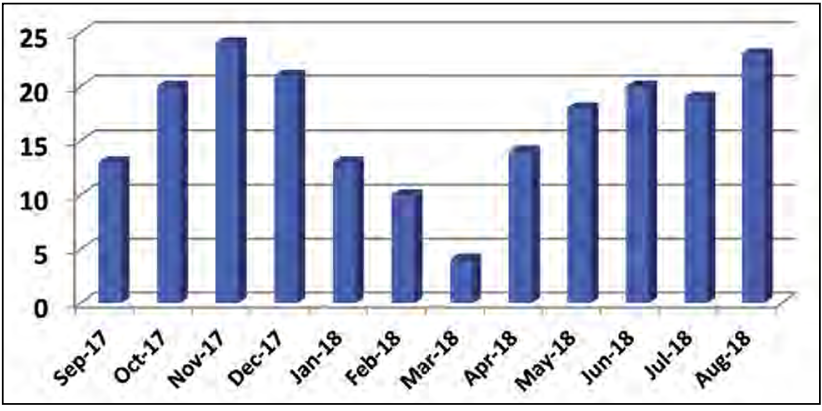

FIGURE 1. Distribution of the number of patients throughout the year

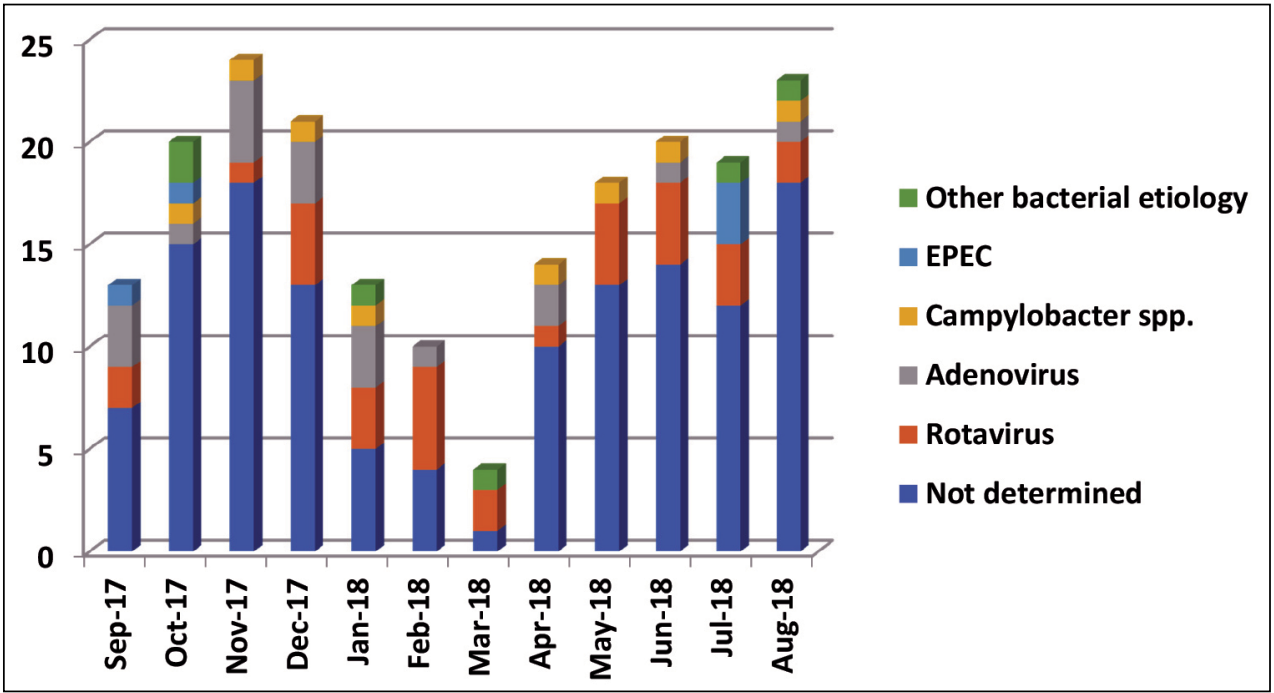

FIGURE 2. Etiological distribution in the studied months 
Rotavirus was found in 15.6\% (31/199) of the patients; adenovirus in 9.5\% (19/199); Campylobacter spp. in 4 \% (8/199), Salmonella spp. and Klebsiella spp. each in 2 patients (each representing 1\%), enteropathogenic Escherichia coli (EPEC) in 2.5\% (5/199) of the patients. In more than half of the patients (65.3\%) (133/199), the etiology of the AGE was not determined. Rotavirus was found to be the dominating confirmed pathogen in most of the months (figure 2).

The mean age \pm SD of the 31 patients with Rotavirus infection was $1.9 \pm 1.8$ years with a median of 1.16 years. The mean age \pm SD of the 19 patients with adenovirus AGE was $2.75 \pm 1.9$ years with a median age of 2.58 years. There was no statistically significant difference regarding the age of the patients with rotavirus and adenovirus $(\mathrm{p}=0.06)$.

The duration of the symptoms from the onset of the disease until discharge ranged between 2-21 days, mean value $\pm \mathrm{SD}=5.2 \pm 2.6$ days, and the median val$\mathrm{ue}=4$ days. One hundred twenty-five out of 199 patients needed IV rehydration $(62.8 \%)$, while 25 patients received antibiotic treatment.

More than half of the patients had signs of moderate dehydration (53.3\%-106/199 patients) and 39.6\% (79/199) signs of mild dehydration. Signs of severe dehydration (1.5\%-3/199 children) or hypovolemic shock $(0.5 \%)$ were rare. As shown in figure 3 , rotavirus caused the highest number of total dehydrationmoderate dehydration in particular.

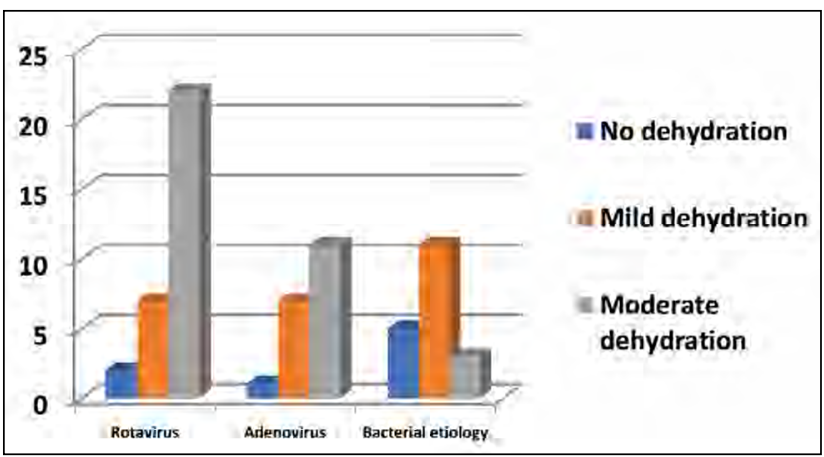

FIGURE 3. Grade of dehydration depending on the etiology

More than half of the patients (51.75\%) had metabolic acidosis (compensated or uncompensated). The acid-base balance was normal in 50 patients $(25.1 \%)$ and was not determined in 38 patients $(19.1 \%)$.

Hyponatremia was present in $21.1 \%$ of the children and hypoglycemia in $7.03 \%$ of them.

\section{DISCUSSIONS}

Our study's results were in line with the literature, with children between 1-5 years is the most common age group affected by AGE. Rotavirus was the most common etiology detected, followed by adenovirus. Important to mention that in two-third of the cases, the etiology couldn't be detected. The dominant months were November followed by August. Additionally, there wasn't a significant difference regarding a predominance of viral or bacterial etiology in certain months or seasons of the year.

In the study conducted by Chung and collaborates (12) regarding the seasonal distribution of the different pathogens, the peak months of rotavirus and Norovirus were April and March respectively in contrast to our results where the peak month for rotavirus was February.

Forty to $60 \%$ of diarrheal cases remain without identified etiology. As diagnostic and identification techniques become more advanced and sophisticated, the percentage of idiopathic diarrheal diseases will decline correspondingly (1).

Dehydration represented the most common complication, mainly mild to moderate, while severe dehydration or hypovolemic shock was rather rare. Regarding acid-base imbalance, more than half of the patients associated metabolic acidosis, mostly a compensated form. Other complications included hyponatremia and hypoglycemia. According to the World Health Organization (WHO) (13): "Diarrheal disease is the second leading cause of death in children under five years old. It is both preventable and treatable." Death is usually due to loss of water and electrolytes, leading to severe dehydration.

Studies of hospitalized children concluded that the AGE cases with detected rotavirus were more severe than the ones in which rotavirus wasn't detected $(14,15,16)$. This includes more severe dehydration, higher vomiting incidences, and increased necessity of parenteral rehydration (4).

Due to the limited resources in low-income countries and the paucity of effective laboratory analysis, the recognition of the clinical signs of dehydration remains a crucial factor in preventing morbidity and mortality, and introduction of early treatment. In their study, Dastidar and collaborators (17) aimed to outline the spectrum of electrolyte disturbances and correlate the parameters with the clinical picture. Their enrolled study population included 200 children, and their findings show $83 \%$ moderately dehydrated children. Based on their study, isonatremic dehydration is the most common electrolyte disturbance found in AGE, followed by hyponatremic dehydration. The latter is more common in children receiving inadequately diluted oral rehydration solutions (ORS). Increasing awareness concerning the proper handling and preparation of ORS is a key solution in helping to 
prevent hyponatremia in children suffering from AGE $(17,18)$.

When comparing rotavirus with non-rotavirus AGE, according to Karampatsas et al. (19), presentations with metabolic acidosis and fever were more frequent in children infected with rotavirus, hence needing hospitalization more than children with AGE due to causes other than rotavirus. An interesting finding was encephalopathy, occurring only in rotavirus infections. Whether caused by rotavirus or other pathogens, the most commonly encountered extraintestinal manifestations were neurological complication. Karampatsas and collaborates (19) concluded that AGE due to rotavirus has a more prolonged and more severe clinical course with surprisingly neurological signs and seizures were frequently encountered. Rotavirus vaccines may successfully decrease these manifestations. In our patients, signs encephalitis were not described.

AGE imposes a remarkable financial burden upon healthcare systems and households, thus risking consequent impoverishment amidst families seeking medical care (20). Therefore, despite the rotavirus vaccine, diarrheal disease among children remains a notable burden, especially in low-income countries. The rotavirus vaccine managed to decrease the number of dehydrated children, hospital admissions, calls, and visits owing to AGE caused by rotavirus (21). The live- attenuated oral monovalent rotavirus vaccine was proven to be highly effective, providing $85 \%$ protection against severe AGE caused by rotavirus and $100 \%$ protection against the most severe episodes of dehydration $(21,22)$.

At the moment, the treatment of AGE is limited to infection control and symptomatic management, by treating and preventing dehydration; no disease-modifying interventions currently exist.

\section{REFERENCES}

1. Galloway DP, Cohen MB. Infectious Diarrhea. In: Wyllie R, Hyams JS, Kay M, editors. Pediatric Gastrointestinal and Liver Disease. $5^{\text {th }}$ ed. Philadelphia: Elsevier 2015; 38: 441-62.

2. Liu L, Johnson HL, Cousens $S$ et al. Global, regional, and national causes of child mortality: An updated systematic analysis for 2010 with time trends since 2000. Lancet 2012; 379: 2151-61.

3. Walker CLF, Rudan I, Liu L et al. Global burden of childhood pneumonia and diarrhea. Lancet 2013; 381: 1405-16.

4. Guarino A, Bruzzese E. Viral diarrhea. In: Guandalini S, Dhawan A, Branski D, editors. Textbook of Pediatric Gastroenterology, Hepatology and Nutrition. $1^{\text {st }}$ ed. Switzerland: Springer; 2016; 14: 159-70.

5. Lanata CF, Walker CLF, Olascoaga AC et al. Child Health Epidemiology Reference Group of the World Health Organization and UNICEF. Global causes of diarrheal disease mortality in children $<5$ years of age: A systematic review. PLoS One 2013; 8: e72788.

6. Denno DM, Shaikh N, Stapp JR et al. Diarrhea etiology in a pediatric emergency department: A case control study. Clin Infect Dis 2012; 55: 897-904.
We have to mention some limitations of the study. Being a retrospective study, the data in the patients' files might be incomplete. Also, we did not take into consideration the vaccination status because it was not mentioned in all the files. The patients who have pathological products in the stool at presentation are not admitted in this clinic. Last but not least in our laboratory analysis, only rotavirus and adenovirus can be detected from a stool sample.

\section{CONCLUSIONS}

In our study, AGE affected children between 1 and 5 years of age primarily. Rotavirus was the leading etiology found in most pediatric age groups in all 12 months of the year. More than half of the children admitted with AGE had complications, but mainly mild or moderate dehydration. The number of patients in which the etiology was not determined is still high, despite the new techniques introduced for determination of the viral antigens in the stool.

Conducting our study was needed and beneficial in order for us as local doctors to get familiar with what we are dealing with in our "backyard", in order to approach, treat and manage this disease in a more efficient way.

Future studies could aim to find objective data regarding the effects of vaccination in Romania in reducing the number of infections and the severity of complications. It would be useful to do prospective studies with clear study design, protocols of investigations, and treatment applied in the same way for all the patients. In order to have a complete picture of the etiology, patients with pathological products in the stool, who are more likely to have bacterial infections, should also be included in the study, in collaboration with the Infectious Disease Department.

7. Centers for Disease Control and Prevention. Rotavirus surveillance worldwide, 2009. MMWR Morb Mortal Wkly Rep 2011; 60: 514-6.

8. Forster J, Guarino A, Parez N et al. Rotavirus Study Group. Hospital-based surveillance to estimate the burden of rotavirus gastroenteritis among European children younger than 5 years of age. Pediatrics 2009; 123: 393-400.

9. Guarino A, Winter H, Bhupinder S, Quak SH, Lanata C. Acute gastroenteritis disease: report of the FISPGHAN Working Group. J Pediatr Gastroenterol Nutr 2012; 55:621-626.

10. Kosek M, Bern C, Guerrant RL. The magnitude of the global burden of diarrheal disease, as estimated from studies published between 1992 and 2000. Bull World Health Organ 2003; 81:197-204.

11. Payne DC, Vinjé J, Szilagyi PG et al. Norovirus and Medically Attended Gastroenteritis in U.S. Children. N Engl J Med 2013; 368:1121-30.

12. Chung N, Wang SM, Shen CF et al. Clinical and epidemiological characteristics in hospitalized young children with acute gastroenteritis in southern Taiwan: According to major pathogens. Journal of 
Microbiology, Immunology and Infection 2017; 50: 915-922.

13. World Health Organization. Diarrhoeal Disease [Internet]. Geneva: World Health Organization; 2017 May. [cited 2019 May 9]. Available from: http://www.who.int/en/news-room/fact-sheets/detail/diarrhoealdisease.

14. Kawada J, Arai N, Nishimura $\mathrm{N}$ et al. Clinical characteristics of norovirus gastroenteritis among hospitalized children in Japan. Microbiol Immunol 2012; 56: 756-9.

15. Junquera CG, de Baranda CS, Mialdea OG, Serrano EB, SánchezFauquier A. Prevalence and clinical characteristics of norovirus gastroenteritis among hospitalized children in Spain. Pediatr Infect Dis J 2009; 28: 604-7.

16. Gonzalez-Galan V, Sanchez-Fauqier A, Obando I et al. High prevalence of community-acquired norovirus gastroenteritis among hospitalized children: A prospective study. Clin Microbiol Infect 2011; 17: 1895-9.

17. Dastidar RG, Konar N. A Study of Electrolyte Disturbances in a Child Presenting with Acute Gastroenteritis, with Special Emphasis on
Hyponatremic Dehydration - A Hospital Based Cross-Sectional Study. Pediatr Ther 2017; 7(2).

18. Hanna M, Saberi MS. Incidence of hyponatremia in children with gastroenteritis. Pediatr Nephrol 2010; 25:1471-5.

19. Karampatsas K, Osborne L, Seah ML, Tong CYW, Prendergast AJ. Clinical characteristics and complications of rotavirus gastroenteritis in children in east London: A retrospective case-control study. PLoSOne. 2018; 13(3): e0194009.

20. Hendrix N, Bar-Zeev N, Atherly D et al. The economic impact of childhood acute gastroenteritis on Malawian families and the healthcare system. BMJ Open 2017, 7:1-5.

21. Hungerford D, Read JM, Cooke RP et al. Early impact of rotavirus vaccination in a large paediatric hospital in the UK. $J$ Hosp Infect 2016; 93: 117-20.

22. Ruiz-Palacios GM, Perez-Schael I, Velazquez FR et al. Human rotavirus vaccine study group. Safety and efficacy of an attenuated vaccine against severe rotavirus gastroenteritis. N Engl J Med 2006; 354: 11-22. 